\title{
Predictors for Dilated Aorta in Repaired and Unrepaired Tetralogy of Fallot
}

\author{
Khaled R. Abd El Meguid1, Hesham B. Mahmoud1, Mostafa M. Mohammad² \\ ${ }^{1}$ Division of Cardiology, Benisuef University Hospital, Benisuef, Egypt \\ ${ }^{2}$ Division of Cardiology, National Heart Institute, Cairo, Egypt \\ Email: Krefaat20@hotmail.com
}

Received 16 January 2015; accepted 23 August 2015; published 26 August 2015

Copyright (C) 2015 by authors and Scientific Research Publishing Inc.

This work is licensed under the Creative Commons Attribution International License (CC BY).

http://creativecommons.org/licenses/by/4.0/

(c) (i) Open Access

\section{Abstract}

Aortic root pathology has been described in patients with Tetralogy of Fallot, although the most common reason for repeat surgery in the adult after TOF repair relates to problems in the right ventricular outflow tract, the aortic root is often forgotten. Objective: We sought to determine those patients with known Fallot tetrallogy at risk for progressive dilatation of the thoracic aorta and explore the common predictors present in this patient group. Methods and Results: A multicenter observational study which enrolled 100 patients $(50$ surgically repaired and 50 before surgical repair of TOF) with standardized reassessment of echocardiographic parameters and multislice CT angiography of the heart and great vessels data. The data were reviewed and analyzed according to the demographic, morphological, surgical and clinical details. We used standard nomograms and $\mathrm{Z}$ score for aortic root dimensions at the level of aortic annulus, sino-tubular junction and sinus of Valsalva based on body surface area. For surgically repaired patients, all the measured diameters across aortic annulus, STJ \& sinus of Valsalva were larger in the dilated unrepaired group with mean \& median of $24.63(3.99) \& 25(15-35), 27.2$ (4.26) \& 27 (17 - 40), 35.97 (4.59) \& $36(24-45) \mathrm{mm}$ respectively compared to a mean \& median of $13.2(2.62) \& 13(9-17)$, $14.53(2.90) \& 14(10-19), 20.53(3.40) \& 21(14-25) \mathrm{mm}$ respectively in the not dilated unrepaired group with significant statistical difference ( $p$ value $<0.0001)$. Also $\mathrm{Z}$ score among unrepaired dilated TOF patients was larger in comparison to the non dilated unrepaired group with significant statistical difference ( $p$ value $<\mathbf{0 . 0 0 0 1 )}$ ). For unrepaired patients, all the measured diameters across aortic annulus, STJ \& sinus of Valsalva were larger in the dilated unrepaired group with mean \& median of 24.63 (3.99) \& 25 (15 - 35), 27.2 (4.26) \& 27 (17 - 40), 35.97 (4.59) \& 36 (24 - 45) mm respectively compared to a mean \& median of $13.2(2.62) \& 13(9-17), 14.53(2.90)$ \& $14(10$ - 19), $20.53(3.40) \& 21(14-25) \mathrm{mm}$ respectively in the not dilated unrepaired group with significant statistical difference ( $p$ value $<0.0001$ ). Also $\mathrm{Z}$ score among unrepaired dilated TOF patients at the level of annulus, STJ \& sinus of Valsalva was larger in comparison to the non dilated unrepaired group with significant statistical difference (p value $<0.0001$ ). Conclusions: The first important finding of this study is the occurrence of significant aortic root dilatation in 
$22 \%$ of patients after intra-cardiac repair of TOF. Older age at repair, long shunt to repair interval and residual ventricular septal defect are the most common variables associated with aortopathy and aortic regurgitation in such group of patients. The second important finding is the occurrence of aortic root dilatation in $70 \%$ of patients before surgical repair of TOF; whereas male sex and TOF with pulmonary atresia appeared to be the most common variables associated with aortopathy and aortic regurgitation in this group of patients.

\title{
Keywords
}

\author{
Tetralogy of Fallot, Aortopathy, Ventricular Septal Defect, Aortic Regurgitation, Pulmonary \\ Regurgitation, Pulmonary Atresia
}

\section{Introduction}

Tetralogy of Fallot (TOF) is the most commonly encountered cyanotic congenital heart defect in infancy, with a frequency of nearly $10 \%$ of all congenital heart disease [1]. Over the last decade, there has been accumulating body of published data documenting progressive aortic dilatation in patients with repaired and unrepaired congenital cardiac defects [2]-[6]. The development and refinements of surgical repair techniques for TOF in the last 40 years have contributed to a steadily increasing population of adult survivors [7]. Factors that may contribute to late morbidity in this cohort of patients and the need for further reintervention include residual right ventricular outflow tract obstruction, right ventricular dysfunction resulting from severe pulmonary regurgitation, tricuspid regurgitation from right ventricular dilatation, residual ventricular septal defect, development of atrial or ventricular tachyarrhythmia and the often insidious development of progressive aortic root dilatation leading to aortic regurgitation and with time left ventricular dysfunction. Although aortic root dilatation has been reported in patients with TOF even after reparative surgery its underlying pathophysiology remains elusive [8] [9]. Progressive aortic root dilatation was thought to be due to increased aortic flow from right to left shunting in TOF patients before surgical repair [8]-[10]. However, there has been some evidence of late progressive aortic root dilatation in a subset of TOF patients even after total corrective surgery. Aim of this work is to outline patients with known Fallot Tetralogy at risk for progressive dilatation of the thoracic aorta and explore the common predictors present in this group of patients.

\section{Material and Methods}

The study reviewed echocardiographic and multislice CT angiography of the heart and great vessels data obtained from 100 patients with known Fallot Tetralogy associated with aortopathy attending the outpatient congenital cardiac clinic at Beni-Suef University Hospital and National Heart Institute. The data were reviewed and analyzed according to the demographic, morphological, surgical and clinical details. Patients were excluded if they had coexisting complex congenital abnormalities (single ventricle physiology or transposition of the great arteries) and connective tissue disorder. No case had prior aortic root or valve repair [11].

\subsection{Data Collection}

Patient charts were reviewed for age, sex, body surface area (BSA), degree of cyanosis as evaluated with pulse oximetry, prior interventions, age at intervention and duration of follow up after intervention, and residual cardiac lesions.

\subsection{Echocardiography}

Aortic root size was measured from M-mode or 2D echo according to American Society of Echocardiography guidelines [12]. Echocardiographic parameters were reviewed and reassessed with a standardized protocol and uniform definitions [13]. Left ventricular (LV) internal dimensions (diastole and systole) were determined in parasternal long-axis views. Color, continuous wave, and pulse-wave Doppler data for all valves were recorded. 
Aortic valve regurgitation was quantified as (0) absent, (1) mild (regurgitant jet width/LV outflow tract diameter $<30 \%$, no flow reversal in the descending aorta, pressure half-time $>400 \mathrm{~ms}$ ), (2) moderate (regurgitant jet width/LV outflow tract diameter, 30\% - 59\%; early flow reversal in descending aorta or pressure half-time, 251 $\mathrm{ms}$ - $399 \mathrm{~ms}$ ), or (3) severe (regurgitant jet width/LV outflow tract diameter $>60 \%$; holodiastolic reversal in descending aorta or pressure half-time $<250 \mathrm{~ms}$ ) [14]. The degree of tricuspid regurgitation was graded as mild, moderate, or severe on the basis of the ratio of the area of the tricuspid regurgitant jet to that of the right atrium; [15]. The severity of pulmonary regurgitation was quantified by the ratio of the regurgitant color jet width to the diameter of the right ventricular outflow tract [16]. The degree of right ventricular outflow obstruction was estimated by continuous wave Doppler assessment [17].

\subsection{Diagnostic Value of 64 Multislice CT in Typing of Congenital Heart Defects Associated with Aortopathy}

MSCT was performed with a 64-MDCT scanner (Siemens, somatom, dual source 64, rotation time 0.33 sec, temporal resolution 80 - 83 msec, Germany). MSCT images were analyzed retrospectively, slice thickness 0.5 $\mathrm{mm}$, overlap $0.3 \mathrm{~mm}$. Increment during data acquisition: $1.5 \mathrm{~mm}$ Multiplanar reconstruction (MPR), curvedplanar reconstruction (CPR), volume render (VR), maximum intensity projection (MIP) and minimum intensity projection (MinIP) were performed in all patients. Intravenous injection of $11 \mathrm{ml}$ of iodixanol 370 through an antecubital vein at a high flow rate $\{2.5 \mathrm{ml} / \mathrm{sec}$. $\}$ was done. An $80 \mathrm{kv}$ was selected in patients less than one year old for CT examination to reduce radiation exposure [18]. MSCT Images were interpreted guided by the anatomical and segmental/sequential approach [19]. Coronal views were reconstructed through the aortic root at the level of annulus, sinus of Valsalva and STJ. The maximal diameters in diastolic phase were assessed [20]. The external diameters were measured perpendicular to the axis of blood flow [21]. These measurements were made by a single observer, associated professor of radiology and analyzed as Z scores based on body surface area. Z scores were calculated from MDCT derived aortic root diameters cited by Detroit [22]. Degree of aortic overriding, arch side and MAPCAS were assessed. Patients were defined as having "aortic root dilatation" if they had measurements corresponding to Z-scores $>3$ at the annulus and aortic sinus, and $>2.5$ at the sino-tubular junction level [23].

\subsection{Statistical Analysis}

Data analysis was performed using STATA intercooled version 9.2. Quantitative data were analyzed using student t-test to compare means of two groups. When the data were not normally distributed Mann-Whitney test was used. Qualitative data were compared using either Chi square test or Fisher exact test. Statistical significance was inferred if p-value was less than 0.05 .

\section{Results}

The current study is an observational analysis of Tetralogy of Fallot patients. The study was conducted in Benisuef University Hospital and National Heart Institute with the aim to identify predictors for aortic root dilatation in Tetralogy of Fallot (ToF) patients. The current study enrolled 100 patients (50 surgically repaired and 50 before surgical repair of TOF).

\subsection{Patients' Population Results}

\subsubsection{Group (1): Surgically Corrected Tetralogy of Fallot Patients}

This group include 50 patients, their mean age is 164.48 (78.04) months \& median (range) 156 (36 - 325) months, with male predominance 64\% (no = $32 \mathrm{pt}$ ), their mean height \& weight is $137.7(27.27) \mathrm{cm} \& 40.99$ (17.56) kg respectively with median 146.5 (83 - 179) cm \& 40 (14 - 72) kg (Table 1). Conduit repair of pulmonary atresia was done in $4 \%$ (no $=2 \mathrm{pt}$ ). Left aortic arch was predominant in $70 \%$ (no $=35 \mathrm{pt}$ ), surgery for absent pulmonary valve was founded in $2 \%$ representing a single case (Table 2). The mean BSA was $1.23(0.39)$ with median $1.26\left(0.55\right.$ - 1.89) $\mathrm{m}^{2}$, their mean $\mathrm{O}_{2}$ saturation was 91.52 (3.19\%) with median $91.5(84 \%$ - 98\%) (Table 3). Their mean, age at repair, shunt duration, repair duration, shunt repair interval was 73.1 (42.86), 143.1 (57.79), 91.38 (49.99), 41.7 (27.84) months respectively with median of 60 (12 - 186), 129 (72 - 264), 84 (12 - 210), 33 (12 - 108) months respectively. Post operative residual VSD was demonstrated in $8 \%$ (no = 4 pt) 
Table 1. Demographic characteristics of repaired TOF patients in study population.

\begin{tabular}{cc}
\hline Characteristics & No. (\%) \\
Age & $10(20.00)$ \\
$<96$ months & $40(80.00)$ \\
$\geq 96$ months & \\
Sex & $18(36.00)$ \\
Female & $32(64.00)$ \\
Male & \\
Height & $137.7(27.27)$ \\
Mean (SD) & $146.5(83-179)$ \\
Median (range) & \\
Weight & $40.99(17.56)$ \\
Mean (SD) & $40(14-72)$ \\
Median (range) &
\end{tabular}

Table 2. Aortic arch side and associated disease in surgically repaired TOF patients.

\begin{tabular}{|c|c|}
\hline Characteristics & No. (\%) \\
\hline \multicolumn{2}{|c|}{ Conduit repair of pulmonary Artesia } \\
\hline No & $48(96)$ \\
\hline Yes & $2(4)$ \\
\hline \multicolumn{2}{|l|}{ Arch side } \\
\hline Left & $35(70.00)$ \\
\hline Right & $15(30.00)$ \\
\hline \multicolumn{2}{|l|}{ Associated disease } \\
\hline No & $49(98.00)$ \\
\hline Absent pulmonary valve & $1(2.00)$ \\
\hline
\end{tabular}

Table 3. BSA and $\mathrm{O}_{2}$ saturation in surgically repaired TOF patients.

\begin{tabular}{cc}
\hline Characteristics & No. (\%) \\
BSA (m²) & \\
Mean (SD) & $1.23(0.39)$ \\
Median (range) & $1.26(0.55-1.89)$ \\
$\mathrm{O}_{2}$ saturation (\%) & \\
Mean (SD) & $91.52(3.19)$ \\
Median (range) & $91.5(84-98)$ \\
$\mathrm{O}_{2}$ saturation (\%) & \\
$<80$ & $0(0.00)$ \\
$\geq 80$ & $50(100.00)$
\end{tabular}

(Table 4). The echo data revealed mean LVEF\% was 60.68 (5.53) with median 62 (48 - 70), less than moderate AR was present in $16 \%$ (no = 8 pt) \& up to severe PR was present in 52\% (no = 26 pt), the mean residual gradient across the RVOT was 22.68 (11.66) mmHg with median 20 (6 - 62) mmHg and mean PASP was 47.4 (14.55) $\mathrm{mmHg}$ with median of 44 (25 - 93) mmHg (Table 5). The mean diameter of the aortic annulus, STJ \& Sinus of valsalva was 20.72 (4.31), 22.96 (4.65) \& 30.14 (6.13) mm respectively with a median of 20.5 (13 - 29), 22.5 (14 - 33) \& 29.5 (18 - 42) mm respectively (Figure 1). Mean/median Z score for the aortic root of this group of patients was $2.21(1.17) / 2.33(0.08-4.45), 1.80(0.89) / 1.80(0.89)$ \& $2.65(1.04) / 2.7(0.4-4.98) \mathrm{mm}$ at the level of aortic annulus, STJ \& Sinus of valsalva respectively (Figure 2). All of them (no $=50$ ) had no residual MAPCS and or PDA after repair. Aortic root dilation was present in $22 \%$ (no = 11 pt) of repaired TOF while $78 \%$ (no = 39 pt) their aortic roots were not dilated. 


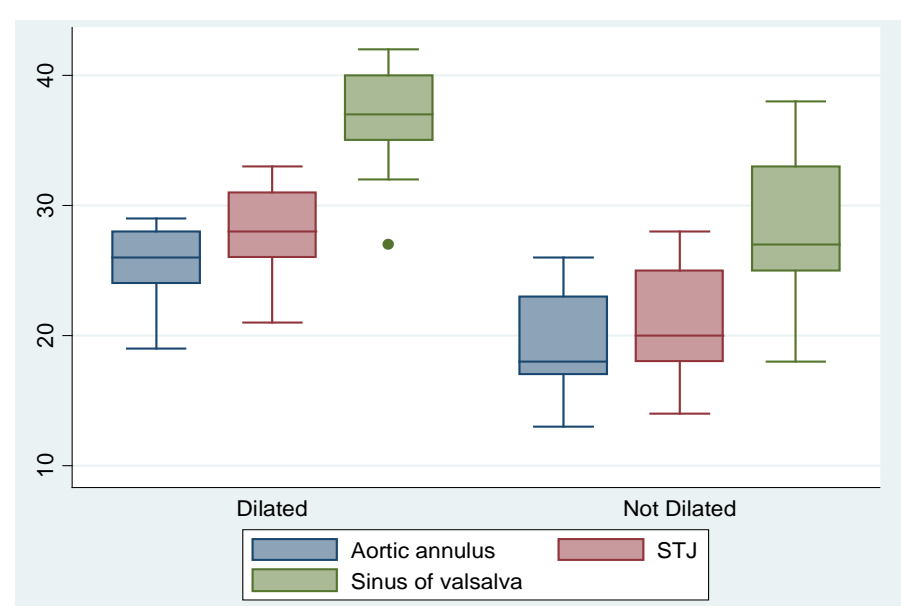

Figure 1. Comparison between patient with dilatation and those without dilation in surgically repaired patients as regard MDCT measurement of study population.

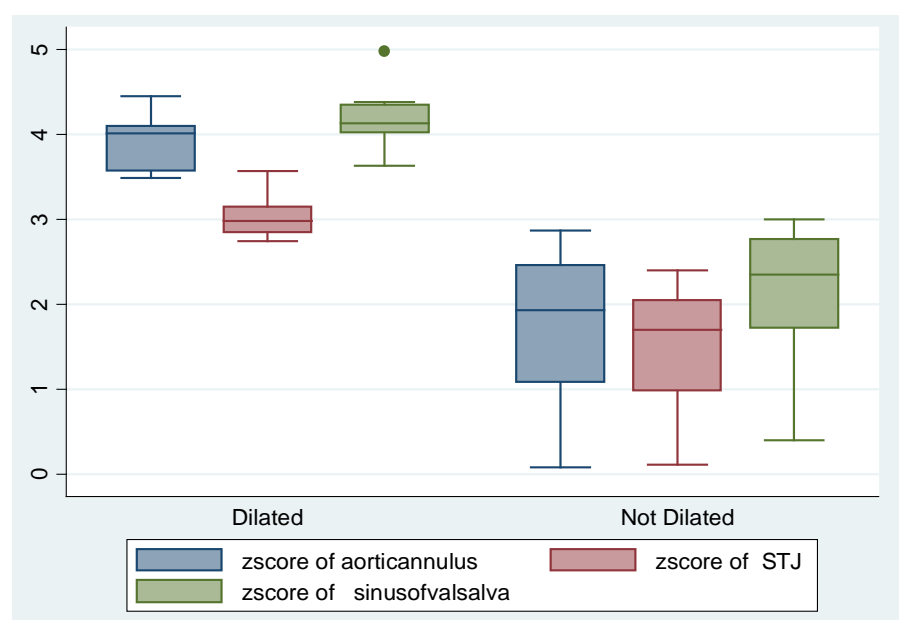

Figure 2. Comparison between repaired TOF patients with dilatation and those without dilation as regard $\mathrm{Z}$ score of study population.

Table 4. Operative criteria of surgically repaired TOF patients.

\begin{tabular}{cc}
\hline Characteristics & No. (\%) \\
\hline Age at repair (months) & $73.1(42.86)$ \\
Mean (SD) & $60(12-186)$ \\
Median (range) & \\
Shunt duration (months) & $143.1(57.79)$ \\
Mean (SD) & $129(72-264)$ \\
Median (range) & \\
Repair duration (months) & $91.38(49.99)$ \\
Mean (SD) & $84(12-210)$ \\
Median (range) & \\
Shunt repair interval (months) & $41.7(27.84)$ \\
Mean (SD) & $33(12-108)$ \\
Median (range) & \\
Residual VSD shunt & $46(92.00)$ \\
No & $4(8.00)$ \\
Yes &
\end{tabular}


Our repaired tetrallogy of Fallot cases were further divided into two subgroups as regard presence of aortic root dilatation defined as aortic root Z score $>3$ at the level of aortic annulus, sinus of Valsalva $\&>2.5$ at the level of STJ (Table 6). Group A1; (Dilated) include 11 patients, their mean \& median age was 189.27 (85.33) \& 174 (60 - 324) months respectively. The mean \& median height was 144.18 (23.36) \& 149 (105 - 175) cm respectively with mean \& median weight in $\mathrm{kg} 45.64$ (17.71) \& 45 (18 - 72) respectively. Eight patients were

Table 5. Echo findings of surgically repaired TOF patients.

\begin{tabular}{cc}
\hline Characteristics & No. (\%) \\
LVEF\% & \\
Mean (SD) & $60.68(5.53)$ \\
Median (range) & $62(48-70)$ \\
AR & \\
No & $42(84.00)$ \\
Yes & $8(16.00)$ \\
PR & \\
No & $24(48.00)$ \\
Yes & $26(52.00)$ \\
RVOT obstruction & \\
Mean (SD) & $22.68(11.66)$ \\
Median (range) & $20(6-62)$ \\
RVOT obstruction & \\
$<25$ mmhg & $33(66.00)$ \\
$\geq 25$ mmhg & $17(34.00)$ \\
PASP mmhg & \\
Mean (SD) & $47.4(14.55)$ \\
Median (range) & $44(25-93)$ \\
\hline
\end{tabular}

Table 6. Aortic root dimensions and corresponding Z score derived from MDCT of surgically repaired TOF patients.

\begin{tabular}{|c|c|}
\hline Characteristics & No. (\%) \\
\hline $\begin{array}{l}\text { aortic annulus } \\
\text { Mean (SD) } \\
\text { Median (range) }\end{array}$ & $\begin{array}{c}20.72(4.31) \\
20.5(13-29)\end{array}$ \\
\hline $\begin{array}{c}\text { Z score } \\
\text { Mean (SD) } \\
\text { Median (range) }\end{array}$ & $\begin{array}{c}2.21(1.17) \\
2.33(0.08-4.45)\end{array}$ \\
\hline $\begin{array}{c}\text { STJ } \\
\text { Mean (SD) } \\
\text { Median (range) }\end{array}$ & $\begin{array}{c}22.96(4.65) \\
22.5(14-33)\end{array}$ \\
\hline $\begin{array}{c}\text { Z score } \\
\text { Mean (SD) } \\
\text { Median (range) }\end{array}$ & $\begin{array}{c}1.80(0.89) \\
1.87(0.11-3.57)\end{array}$ \\
\hline $\begin{array}{l}\text { Sinus of valsalva } \\
\text { Mean (SD) } \\
\text { Median (range) }\end{array}$ & $\begin{array}{c}30.14(6.13) \\
29.5(18-42)\end{array}$ \\
\hline $\begin{array}{c}\text { Z score } \\
\text { Mean (SD) } \\
\text { Median (range) }\end{array}$ & $\begin{array}{c}2.65(1.04) \\
2.7(0.4-4.98)\end{array}$ \\
\hline $\begin{array}{c}\text { MAPCS/PDA } \\
\text { No } \\
+\end{array}$ & $\begin{array}{c}50(100.00) \\
0(0.00)\end{array}$ \\
\hline $\begin{array}{l}\text { Conclusion } \\
\text { Dilated } \\
\text { Not dilated }\end{array}$ & $\begin{array}{l}11(22.00) \\
39(78.00)\end{array}$ \\
\hline
\end{tabular}


males representing 72.73\% (Table 7). Group A2; (Non-dilated) include 39 patients, their mean \& median age was 157.49 (75.55) \& 156 (36 - 312) months respectively. The mean \& median height was 135.87 (28.29) \& 132 (83 - 179) cm respectively with mean \& median weight 39.67 (17.51) \& 35 (14 - 71) Kg respectively, Twenty four patients were males representing $61.54 \%$. There was no statistical significant difference between the two groups according to their age and sex (Table 7). Two patients (18.18\%) among dilator group had conduit repair of pulmonary atresia while none of the patients among the non dilator which was statistically significant. Right arch was diagnosed in 54.55\% among dilator group (no $=6 \mathrm{pt}$ ) while the right arch was founded in 23.08\% (no $=9 \mathrm{pt}$ ) among the non dilator which was statistically significant. Only one patient had surgical intervention for absent pulmonary valve among the dilated group (Table 8). The mean \& median BSA in the dilated \& in non-dilated groups were $1.33(0.38), 1.20(0.40), 1.37(0.71-1.87) \& 1.16(0.55-1.89) \mathrm{m}^{2}$ respectively. Both groups had $\mathrm{O}_{2}$ saturation $>80 \%$ but the dilated group had significant lower mean 89 (3.41) \& median 88 (84 - 95) $\mathrm{O}_{2}$ saturation in comparison to non-dilated group with mean \& median $\mathrm{O}_{2}$ saturation of 92.23 (2.79) \& 92 (86 - 98) respectively (Table 9). The repaired dilator TOF patients showed statistically significant older age at repair with mean \& median age of 112.36 (44.50) \& 126 (36 - 186) months respectively while the non-dilated group had a mean \& median age at repair of 62.03 (35.71) \& 48 (12 - 162) months respectively. There was no statistical significant difference between the two groups as regard shunt $\&$ repair duration. The dilator group had statistical significant longer shunt repair interval with mean duration of 84 (16.97) \& median of 84 (60 - 108) months while the non-dilated group mean \& median shunt repair duration were 27.6 (10.83) \& 24 (12 - 48) months respectively. Residual VSD shunt was present in $36.36 \%$ (no $=4 \mathrm{Pt}$ ) of dilated group and none of the non-dilated group with p value $<0.0001$ (Table 10). Mean \& median LVEF\% showed a statistically significant lower values in repaired dilator TOF patients equal to 56.18 (7.11) \& 58 (48 - 65)\% respectively and a

Table 7. Comparison between patient with Aortic root dilatation and those without dilation in surgically repaired TOF patients.

\begin{tabular}{cccc}
\hline Characteristics & Dilated & Not dilated & p value \\
\hline Age & & & \\
$<96$ months & $1(9.09)$ & $9(23.08)$ & 0.31 \\
$>96$ months & $10(90.91)$ & & \\
Sex & & $15(38.46)$ & 0.50 \\
Female & $3(27.27)$ & & \\
Male & $8(72.73)$ & $135.87(28.29)$ & \\
Height & & $132(83-179)$ & \\
Mean (SD) & $144.18(23.36)$ & & \\
Median (range) & $149(105-175)$ & $39.67(17.51)$ & \\
Weight & & $35(14-71)$ & \\
Mean (SD) & $45.64(17.71)$ & & \\
Median (range) & $45(18-72)$ &
\end{tabular}

Table 8. Comparison between surgically repaired TOF patients with aortic root dilatation and those without dilation in relation to aortic arch side and associated disease.

\begin{tabular}{cccc}
\hline Characteristics & Dilated & Not dilated & p value \\
\hline $\begin{array}{c}\text { Conduit repair of } \\
\text { pulmonary Artesia } \\
\text { No }\end{array}$ & $9(81.82)$ & $39(100.00)$ & \\
Yes & $2(18.18)$ & $0(0.00)$ & $0.007^{*}$ \\
Arch side & & & \\
Left & $5(45.45)$ & $30(76.92)$ & $0.04^{*}$ \\
Right & $6(54.55)$ & $9(23.08)$ & \\
Tab association & & & \\
No & $10(90.91)$ & 0.06 & \\
Absent pulmonary valve & $1(9.09)$ & $0(0.00)$ & \\
\hline
\end{tabular}


Table 9. Comparison between surgically repaired TOF patients with dilatation and those without dilation in patients as regard BSA and $\mathrm{O}_{2}$ saturation.

\begin{tabular}{cccc}
\hline Characteristics & Dilated & Not dilated & p value \\
\hline BSA & & & \\
Mean (SD) & $1.33(0.38)$ & $1.20(0.40)$ & 0.35 \\
Median (range) & $1.37(0.71-1.87)$ & $1.16(0.55-1.89)$ & \\
O saturation & & & \\
Mean (SD) & $89(3.41)$ & $92.23(2.79)$ & $0.007^{*}$ \\
Median (range) & $88(84-95)$ & $92(86-98)$ & \\
$\mathrm{O}_{2}$ saturation & & & \\
$<80$ & $0(0.00)$ & $0(0.00)$ & \\
$\geq 80$ & $11(100.00)$ & $39(100.00)$ & \\
\hline
\end{tabular}

Table 10. Comparison between repaired TOF patients with dilatation and those without dilation as regard operative criteria.

\begin{tabular}{cccc}
\hline Characteristics & Dilated & Not dilated & p value \\
\hline Age at repair & & & \\
Mean (SD) & $112.36(44.50)$ & $62.03(35.71)$ & $0.001^{*}$ \\
Median (range) & $126(36-186)$ & $48(12-162)$ & \\
Shunt duration & & & 0.27 \\
Mean (SD) & $174(72.50)$ & $132.8(50.75)$ & \\
Median (range) & $192(72-264)$ & $108(84-240)$ & 0.24 \\
Repair duration & & & \\
Mean (SD) & $76.91(54.26)$ & $95.46(48.69)$ & $0.009^{*}$ \\
Median (range) & $60(12-180)$ & $90(15-210)$ & \\
Shunt repair interval & & & \\
Mean (SD) & $84(16.97)$ & $27.6(10.83)$ & \\
Median (range) & $84(60-108)$ & $24(12-48)$ & \\
Residual VSD shunt & & & \\
No & $7(63.64)$ & $0.0001^{*}$ \\
Yes & $4(36.36)$ & $0(0.00)$ & \\
\hline
\end{tabular}

mean \& median of 61.95 (4.32) \& 61.95 (4.32)\% in the non-dilated group. Among repaired dilator TOF patients, there was a statistically significant higher residual RVOT gradient with mean \& median of 33.09 (17.66) \& 27 (15 - 62) mmHg with mean \& median gradient of 19.74 (7.28) \& 19 (6 - 35) mmHg in the repaired non-dilated group. On measuring the PASP, mean \& median values were 61.18 (18.27) and 62 (38 - 93) mmHg among dilated group compared to a mean \& median PASO of 43.51 (10.71) and 43 (25 - 78) mmHg in the non-dilated group with p value $=0.001$. Eight patients $72 \%$ of the dilated group had AR of less than moderate while none of the patients in the non-dilated group showed AR and this was of statistically significant value $(\mathrm{P}<0.0001)$. Pulmonary regurgitation was present in $81 \%$ (no $=9 \mathrm{pt}$ ) of the dilated group compared to $43 \%$ of the non-dilated group and this was also of statistically significant value (p 0.03) (Table 11). All measured aortic diameters including aortic annulus, STJ \& sinus of Valsalva were larger in the dilated group with mean \& median of 25.36 (3.04) 26 (19 - 29), 27.82 (3.49) 28 (21 - 33) \& 36.81 (4.47) 37 (27 - 42) mm respectively compared to the mean \& median of the non-dilated group which were 19.41 (3.68) 18 (13 - 26), 21.59 (3.99) 20 (14 - 28), 28.26 (5.16) 27 (18 - 38) mm respectively with statistically significant difference (Table 12). Also Z score among repaired dilator TOF patients at the level of annulus, STJ \& sinus of Valsalva were larger compared to the non-dilated group with statistically significant difference (Table 12).

\subsubsection{Group (2): Unrepaired Tetralogy of Fallot Patients}

This group included 50 patients before undergoing any reparative surgery, their mean age at presentation was 138.06 (82.04) months \& median (range) 126 (12 - 312) months, with male predominance $70 \%$ (no = 35 pt), their mean height \& weight 123.51 (30.87) cm \& 34.17 (18.41) kg respectively with median 126.5 (61 - 168) cm 
Table 11. Comparison between repaired TOF patients with dilatation and those without dilation regard Echo finding of study population.

\begin{tabular}{|c|c|c|c|}
\hline Characteristics & Dilated & Not dilated & $\mathrm{p}$ value \\
\hline $\begin{array}{c}\text { EF\% } \\
\text { Mean (SD) } \\
\text { Median (range) }\end{array}$ & $\begin{array}{l}56.18(7.11) \\
58(48-65)\end{array}$ & $\begin{array}{l}61.95(4.32) \\
62(55-70)\end{array}$ & $0.03^{*}$ \\
\hline $\begin{array}{l}\text { AR } \\
\text { No } \\
\text { Yes }\end{array}$ & $\begin{array}{l}3(27.27) \\
8(72.73)\end{array}$ & $\begin{array}{c}39(100.00) \\
0(0.00)\end{array}$ & $<0.0001^{*}$ \\
\hline $\begin{array}{l}\text { PR } \\
\text { No } \\
\text { Yes }\end{array}$ & $\begin{array}{l}2(18.18) \\
9(81.82)\end{array}$ & $\begin{array}{l}22(56.41) \\
17(43.59)\end{array}$ & $0.03^{*}$ \\
\hline $\begin{array}{l}\text { RVOT obstruction } \\
\text { Mean (SD) } \\
\text { Median (range) }\end{array}$ & $\begin{array}{c}33.09(17.66) \\
27(15-62)\end{array}$ & $\begin{array}{c}19.74(7.28) \\
19(6-35)\end{array}$ & $0.02^{*}$ \\
\hline $\begin{array}{c}\text { RVOT obstruction } \\
\quad<25 \\
\geq 25\end{array}$ & $\begin{array}{l}5(45.45) \\
6(54.55)\end{array}$ & $\begin{array}{l}28(71.79) \\
11(28.21)\end{array}$ & 0.10 \\
\hline $\begin{array}{c}\text { PASP } \\
\text { Mean (SD) } \\
\text { Median (range) }\end{array}$ & $\begin{array}{c}61.18(18.27) \\
62(38-93)\end{array}$ & $\begin{array}{c}43.51(10.71) \\
43(25-78)\end{array}$ & $0.001^{*}$ \\
\hline
\end{tabular}

Table 12. Comparison between repaired TOF patients with dilatation and those without dilation as regard aortic root dimensions and corresponding $\mathrm{Z}$ score derived from MDCT of study population.

\begin{tabular}{|c|c|c|c|}
\hline Characteristics & Dilated & Not dilated & $\mathrm{p}$ value \\
\hline \multicolumn{4}{|l|}{ aortic annulus } \\
\hline Mean (SD) & $25.36(3.04)$ & $19.41(3.68)$ & \multirow{2}{*}{$0.0001^{*}$} \\
\hline Median (range) & $26(19-29)$ & $18(13-26)$ & \\
\hline \multicolumn{4}{|l|}{ Z score } \\
\hline Mean (SD) & $3.91(0.30)$ & $1.73(0.83)$ & \multirow{2}{*}{$<0.0001^{*}$} \\
\hline Median (range) & $4.01(3.49-4.45)$ & $1.93(0.08-2.87)$ & \\
\hline \multicolumn{4}{|l|}{ STJ } \\
\hline Mean (SD) & $27.82(3.49)$ & 21.59 (3.99) & \multirow{2}{*}{$0.0002^{*}$} \\
\hline Median (range) & $28(21-33)$ & $20(14-28)$ & \\
\hline \multicolumn{4}{|l|}{ Z score } \\
\hline Mean (SD) & $3(0.23)$ & $1.46(0.69)$ & \multirow{2}{*}{$<0.0001^{*}$} \\
\hline Median (range) & $2.98(2.74-3.57)$ & $1.7(0.11-2.4)$ & \\
\hline \multicolumn{4}{|l|}{ Sinus of Valsalva } \\
\hline Mean (SD) & $36.81(4.47)$ & $28.26(5.16)$ & \multirow{2}{*}{$0.0001^{*}$} \\
\hline Median (range) & $37(27-42)$ & $27(18-38)$ & \\
\hline \multicolumn{4}{|l|}{ Z score } \\
\hline Mean (SD) & $4.18(0.35)$ & $2.21(0.69)$ & \multirow{2}{*}{$<0.0001^{*}$} \\
\hline Median (range) & $4.13(3.63-4.98)$ & $2.35(0.4-3)$ & \\
\hline \multicolumn{4}{|l|}{ MAPCS/PDA } \\
\hline No & $11(100.00)$ & 39 (100.00) & \\
\hline+ & $0(0.00)$ & $0(0.00)$ & \\
\hline
\end{tabular}


\& 29.5 (7 - 68) kg (Table 13). Right aortic arch was diagnosed in 28\% (no = 14 pt) while $46 \%$ (no = 23 pt) had DORV and or pulmonary atresia. TOF was associated with Down syndrome in only one case 2\% (Table 14). The mean BSA was $1.05 \mathrm{~m}^{2}(0.42)$ with median $1.01 \mathrm{~m}^{2}(0.32-1.77)$, their mean $\mathrm{O}_{2}$ saturation was $75.46 \%$ (9.13) with median 76\% (48 - 95). $\mathrm{O}_{2}$ saturation less than $80 \%$ was measured in $60 \%$ (no = 30 pt) $($ Table 15$)$. The echo data revealed mean LVEF\% of 59\% (4.86) with median 60\% (48 - 71), trivial to mild AR was present in $18 \%$ (no $=9 \mathrm{pt}$ ) \& mild PR was present in only one case, the mean gradient across the RVOT was 59.16 mmHg (19.50) with median 62.5 mmHg (25 - 91) and mean PASP was 32.68 mmHg (15.35) with median of 27 mmHg (14 - 72). Aortic override $>50$ was demonstrated in 42\% (no = $21 \mathrm{pt)}$ in MDCT imaging (Table 16). The mean diameter of the aortic annulus, STJ \& Sinus of valsalva was 21.2 (6.40), 23.4 (7.03) \& 31.34 (8.31) mm respectively with a median of 23 (9 - 35), 25.5 (10 - 40) \& 34 (14 - 45) mm respectively (Figure 3). Mean/median $\mathrm{Z}$ score for the aortic root of this group of patients was $3.06(1.60) / 3.37$ (0.33 - 6.26), 2.78 (2.89)/2.74 (0.19 - 21) \& $3.63(1.17) / 3.97$ (0.99 5.66) $\mathrm{mm}$ at the level of aortic annulus, STJ \&Sinus of valsalva respectively (Figure 4). MAPCS and or PDA were present in 48\% (no = 24 pt) of unrepaired TOF cases in this study. Aortic

Table 13. Demographic characteristics of TOF patients before surgical repair.

\begin{tabular}{cc}
\hline Characteristics & No. (\%) \\
Age & \\
$<96$ months & $28(56.00)$ \\
$\geq 96$ months & $22(44.00)$ \\
Sex & $15(30.00)$ \\
Female & $35(70.00)$ \\
Male & \\
Height & $123.51(30.87)$ \\
Mean (SD) & $126.5(61-168)$ \\
Median (range) & \\
Weight & $34.17(18.41)$ \\
Mean (SD) & $29.5(7-68)$ \\
Median (range) &
\end{tabular}

Table 14. Arch Side and associated disease of unrepaired TOF.

\begin{tabular}{cc}
\hline Characteristics & No. (\%) \\
\hline Arch side & \\
Left & $36(72.00)$ \\
Right & $14(28.00)$ \\
& \\
Tab association & $24(24.00)$ \\
No & $23(46.00)$ \\
DORV and pulmonary atresia & $1(2.00)$ \\
Down syndrome & \\
\hline
\end{tabular}

Table 15. BSA and $\mathrm{O}_{2}$ saturation of unrepaired TOF patients.

\begin{tabular}{cc}
\hline Characteristics & No. (\%) \\
\hline BSA $\left(\mathrm{m}^{2}\right)$ & \\
Mean (SD) & $1.05(0.42)$ \\
Median (range) & $1.01(0.32-1.77)$ \\
$\mathrm{O}_{2}$ saturation \% & \\
Mean (SD) & $75.46(9.13)$ \\
Median (range) & $76(48-95)$ \\
$\mathrm{O}_{2}$ saturation \% & \\
$<80$ & $30(60.00)$ \\
$\geq 80$ & $20(40.00)$ \\
\hline
\end{tabular}




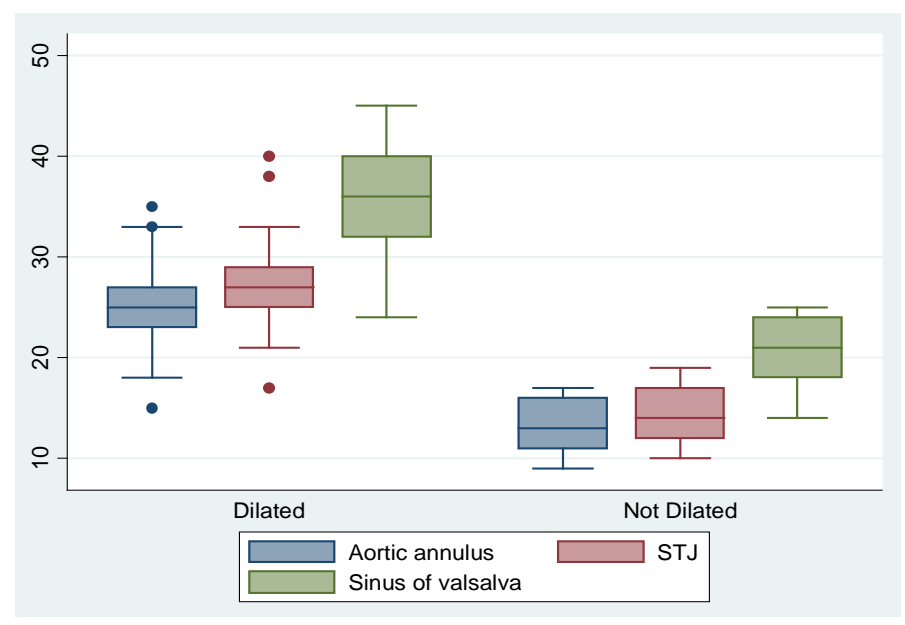

Figure 3. Comparison between unrepaired TOF patients with dilatation and those without dilation as regard MDCT measurement of the aortic root dimensions.

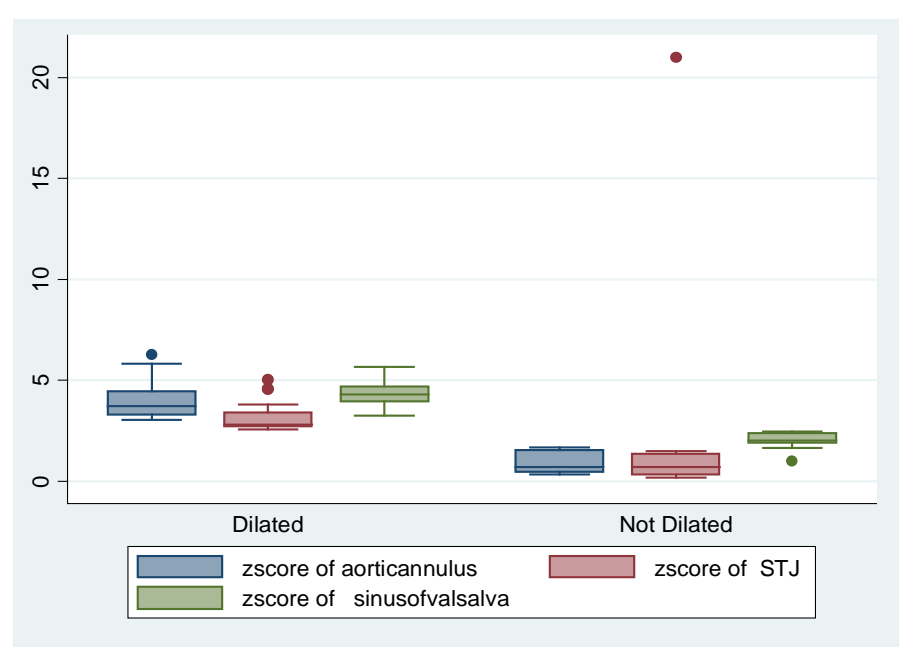

Figure 4. Comparison between unrepaired TOF patients with dilatation and those without dilation as regard aortic root $\mathrm{Z}$ score.

root dilation was present in $70 \%$ (no $=35 \mathrm{pt}$ ) of unrepaired TOF while $30 \%$ (no $=15 \mathrm{pt}$ ) their aortic roots were not dilated. Our unrepaired Tetralogy of Fallot cases were further divided into two subgroups as regard presence of aortic root dilatation defined as aortic root Z score $>3$ at the level of aortic annulus, sinus of Valsalva \& $>2.5$ at the level of STJ (Table 17). Group B1; (Dilated) include 35 patients, their mean \& median age was 172.2 (72.81) \& 180 (36 - 312) months respectively. The mean \& median height was 137.93 (23.53) \& 146 (83 - 168) cm respectively with mean \& median weight of 41.89 (16.45) \& 42 (12 - 68) Kg respectively, thirty patients were males representing 85\% (Table 18). Group B2; (Not dilated) include 15 patients, their mean \& median age was 158.4 (31.13) \& 166 (12 - 108) months respectively. The mean \& median height was 89.87 (16.06) \& 93 (61 - 110) cm respectively with mean \&median weight of 16.17 (5.67) \& 15 (7 - 26) Kg respectively, five patients were males representing 33\% (Table 18). There was statistical significant correlation between male sex and aortic root dilatation among unrepaired TOF cases in our study ( $p$ value $<0.0001$ ) but not with the age at presentation (Table 18). Right arch was diagnosed in 37.14\% among dilated group (no $=13 \mathrm{pt}$ ) while the right arch was founded in $6.67 \%$ (no $=1 \mathrm{pt})$ among the non dilated subgroup which considered statistically significant correlation between right arch and aortic root dilatation ( $\mathrm{p}$ value 0.03 ). Pulmonary atresia and or DORV was founded in \% 62.16 among dilated group (no $=23 \mathrm{pt}$ ) while $0 \%$ among the non dilated subgroup ( $\mathrm{p}$ value $<$ 0.0001) which was highly statistically significant (Table 19). The mean \& median BSA in the dilated \& in non 
Table 16. Echo finding of unrepaired TOF patients.

\begin{tabular}{cc}
\hline Characteristics & No. (\%) \\
LVEF\% & \\
Mean (SD) & $59.3(4.86)$ \\
Median (range) & $60(48-71)$ \\
Aortic Regurgitation & \\
No & $41(82.00)$ \\
Yes & $9(18.00)$ \\
& \\
No & $49(98.00)$ \\
Yes & $1(2.00)$ \\
Pulmonary Regurgitation & \\
RVOT obstruction & $59.16(19.50)$ \\
Mean (SD) & $62.5(25-91)$ \\
Median (range) & \\
RVOT obstruction & $0(0.00)$ \\
$<25$ mmhg & $50(100.00)$ \\
$\geq 25$ mmhg & \\
PASP (mmhg) & $32.68(15.35)$ \\
Mean (SD) & $27(14-72)$ \\
Median (range) & \\
Aortic override & $29(58.00)$ \\
$<50$ & $21(42.00)$ \\
$\geq 50$ &
\end{tabular}

Table 17. MDCT measurement of aortic root \&corresponding $\mathrm{Z}$ scores of unrepaired TOF.

\begin{tabular}{cc}
\hline Characteristics & No. (\%) \\
\hline aortic annulus & $21.2(6.40)$ \\
Mean (SD) & $23(9-35)$ \\
Median (range) & \\
Z score & $3.06(1.60)$ \\
Mean (SD) & $3.37(0.33-6.26)$ \\
Median (range) & \\
STJ & $23.4(7.03)$ \\
Mean (SD) & $25.5(10-40)$ \\
Median (range) & \\
Z score & $2.78(2.89)$ \\
Mean (SD) & $2.74(0.19-21)$ \\
Median (range) & \\
Sinus of valsalva & $31.34(8.31)$ \\
Mean (SD) & $34(14-45)$ \\
Median (range) & \\
Z score & $3.63(1.17)$ \\
Mean (SD) & $3.97(0.99-5.66)$ \\
Median (range) & \\
MAPCS/PDA & $26(52.00)$ \\
No & $24(48.00)$ \\
+ & \\
Conclusion & $35(70.00)$ \\
Dilated & $15(30.00)$ \\
Not dilated &
\end{tabular}


dilated groups was $1.24(0.36) 1.26\left(0.51\right.$ - 1.77) \& $0.61(0.17) \& 0.61\left(0.32\right.$ - 0.85) $\mathrm{m}^{2}$ respectively. The mean \& median $\mathrm{O}_{2}$ saturation among dilated group was 72.97 (9.78) \& 73 (48 - 95)\% and 81.27 (2.74) \& 81 (76 - 86)\% among non dilated unrepaired TOF subgroup. Systemic arterial desaturation $<80 \%$ was measured in $77.14 \%$ (no $=27 \mathrm{pt}$ ) among the dilated group while $20 \%(\mathrm{no}=3 \mathrm{pt}$ ) among the non dilated subgroup. This reflects a significant statistical correlation between systemic arterial desaturation $<80 \%$ and aortic root dilation in unrepaired TOF (p value < 0.0001) (Table 20). There was statistically significant lower mean \& median LVEF\% diagnosed by echo (p value 0.04) in unrepaired dilated TOF patients equal 58.43 (5.28) \& 59 (48 - 71)\% compared to a mean \& median of 61.33 (2.94) \& 61 (55 - 66)\% in the non dilated group (Table 21). Among unrepaired dilated TOF patients there was statistically significant higher RVOT gradient with a mean \& median of 66 (17.78) \& 69 (28 - 91) mmHg compared to 43.2 (13.14) \& 40 (25 - 66) $\mathrm{mmHg}$ in the unrepaired non dilated group (p value 0.0001) (Table 21). The mean \& median PASP pressure was 27.8 (13.68) \& 24 (14 - 72) mmHg among dilated

Table 18. Comparison between unrepaired TOF patients with dilatation and those without dilation as regard demographic characteristics.

\begin{tabular}{cccc}
\hline Characteristics & Dilated & Not dilated & p value \\
\hline Age & & & \\
$<96$ months & $22(62.86)$ & $6(40.00)$ & 0.14 \\
$>96$ months & $13(37.14)$ & $9(60.00)$ & \\
Sex & & & $<0.0001^{*}$ \\
Female & $5(14.29)$ & $10(66.67)$ & \\
Male & $30(85.71)$ & $5(33.33)$ & \\
Height & & & \\
Mean (SD) & $137.93(23.53)$ & $89.87(16.06)$ & \\
Median (range) & $146(83-168)$ & $93(61-110)$ & \\
Weight (kg) & & $16.17(5.67)$ & \\
Mean (SD) & $41.89(16.45)$ & $15(7-26)$ & \\
Median (range) & $42(12-68)$ & & \\
& & &
\end{tabular}

Table 19. Comparison between unrepaired TOF patients with dilatation and those without dilation as regard arch side and associated disease.

\begin{tabular}{cccc}
\hline Characteristics & Dilated & Not dilated & p value \\
\hline Arch side & & & \\
Left & $22(62.86)$ & $14(93.33)$ & $0.03^{*}$ \\
Right & $13(37.14)$ & $1(6.67)$ & \\
Tab association & & & \\
No & $13(35.14)$ & $15(100.00)$ & $<0.0001^{*}$ \\
DORV \& pulmonary atresia & $23(62.16)$ & $0(0.00)$ & \\
Down syndrome & $1(2.70)$ & $0(0.00)$ & \\
\hline
\end{tabular}

Table 20. Comparison between unrepaired TOF patients with dilatation and those without dilation as regard BSA and $\mathrm{O}_{2}$ saturation.

\begin{tabular}{cccc}
\hline Characteristics & Dilated & Not dilated & p value \\
\hline BSA (m²) & & & \\
Mean (SD) & $1.24(0.36)$ & $0.61(0.17)$ & \\
Median (range) & $1.26(0.51-1.77)$ & $0.61(0.32-0.85)$ & \\
& & & \\
$\mathrm{O}_{2}$ saturation \% & & $81.27(2.74)$ & \\
Mean (SD) & $72.97(9.78)$ & $81(76-86)$ & \\
Median (range) & $73(48-95)$ & & $<004^{*}$ \\
$\mathrm{O}_{2}$ saturation \% & & $3(20.00)$ & \\
$<80$ & $27(77.14)$ & $12(80.00)$ & \\
$\geq 80$ & $8(22.86)$ & & \\
\hline
\end{tabular}


Table 21. Comparison between unrepaired TOF patients with dilatation and those without dilation as regard Echo finding.

\begin{tabular}{|c|c|c|c|}
\hline Characteristics & Dilated & Not dilated & $\mathrm{p}$ value \\
\hline $\begin{array}{c}\text { LVEF\% } \\
\text { Mean (SD) } \\
\text { Median (range) }\end{array}$ & $\begin{array}{l}58.43(5.28) \\
59(48-71)\end{array}$ & $\begin{array}{l}61.33(2.94) \\
61(55-66)\end{array}$ & $0.04^{*}$ \\
\hline $\begin{array}{c}\text { Aortic Regurgitatio } \\
\text { No } \\
\text { Yes }\end{array}$ & $\begin{array}{c}26(74.29) \\
9(25.71)\end{array}$ & $\begin{array}{c}15(100.00) \\
0(0.00)\end{array}$ & $0.03^{*}$ \\
\hline $\begin{array}{c}\text { Pulmonary Regurgita } \\
\text { No } \\
\text { Yes }\end{array}$ & $\begin{array}{c}34(97.14) \\
1(2.86)\end{array}$ & $\begin{array}{c}15(100.00) \\
0(0.00)\end{array}$ & 0.51 \\
\hline $\begin{array}{l}\text { RVOT obstructior } \\
\text { Mean (SD) } \\
\text { Median (range) }\end{array}$ & $\begin{array}{c}66(17.78) \\
69(28-91)\end{array}$ & $\begin{array}{l}43.2(13.14) \\
40(25-66)\end{array}$ & $0.0001^{*}$ \\
\hline $\begin{array}{l}\text { RVOT obstructior } \\
\quad<25 \mathrm{mmhg} \\
\geq 25 \mathrm{mmhg}\end{array}$ & $\begin{array}{c}0(0.00) \\
35(100.00)\end{array}$ & $\begin{array}{c}0(0.00) \\
15(100.00)\end{array}$ & $0.02^{*}$ \\
\hline $\begin{array}{l}\text { PASP(mmhg) } \\
\text { Mean (SD) } \\
\text { Median (range) }\end{array}$ & $\begin{array}{l}27.8(13.68) \\
24(14-72)\end{array}$ & $\begin{array}{c}44.07(13.09) \\
39(26-64)\end{array}$ & $0.0001^{*}$ \\
\hline $\begin{array}{c}\text { Aortic override } \\
\quad<50 \\
\geq 50\end{array}$ & $\begin{array}{l}15(42.86) \\
20(57.14)\end{array}$ & $\begin{array}{c}14(93.33) \\
1(6.67)\end{array}$ & $0.001^{*}$ \\
\hline
\end{tabular}

group compared to 44.07 (13.09) \& 39 (26 - 64) mmHg in the non dilated group with (p value $<0.0001$ ) (Table 21). Aortic regurgitation was diagnosed in $25.71 \%$ (no $=9 \mathrm{pt}$ ) of the dilated group, no patient in the not dilated group showed AR and this was of statistically significant correlation (p value 0.03 ) (Table 21). Aortic overriding $>50 \%$ was demonstrated in 57.14\% (no $=20$ pt) among dilated unrepaired compared to $6.67 \%$ among non dilated group which gives a significant statistical correlation between aortic override $>50 \%$ and aortic root dilation (Table 21). All the measured diameters across aortic annulus, STJ \& sinus of Valsalva were larger in the dilated unrepaired group with mean \& median of 24.63 (3.99) \& 25 (15 - 35), 27.2 (4.26) \& 27 (17 - 40), 35.97 (4.59) \& 36 (24 - 45) mm respectively compared to a mean \& median of 13.2 (2.62) \& 13 (9 - 17), 14.53 (2.90) \& 14 (10 - 19), 20.53 (3.40) \& 21 (14 - 25) mm respectively in the not dilated unrepaired group with significant statistical difference ( $\mathrm{p}$ value $<0.0001$ ) (Table 22). Also Z score among unrepaired dilated TOF patients at the level of annulus, STJ \& sinus of Valsalva were larger in comparison to the non dilated unrepaired group with significant statistical difference (p value < 0.0001) (Table 22). MAPCAs and or PDA were founded in 54.29\% (no $=19$ pt) among unrepaired dilators TOF, but none in the unrepaired non dilated TOF group (Table 22).

\section{Discussion}

The current study is an observational study to assess the relationship between clinical characteristics of patients with TOF including age and other variables with aortic root dilatation. Measurements of the aorta were made at the annulus, sinuses and sinotubular junction (STJ) converted to z-scores based on body surface area.

\subsection{Aortic Root Dilatation in Repaired Tetralogy of Fallot}

Surgeries for TOF have increasingly included intervention on the ascending aorta [24]. In operated patients with TOF, the cause of aortic root dilatation is thought to be predominantly secondary to chronic hemodynamic stress from volume overload of the aorta [25]. In 1997, Dodds and colleagues described the first series report about progressive aortic regurgitation and aortic root dilatation after complete, uncomplicated repair of TOF (9). A second study done in 2002 by Niwa K, concluded that long-standing volume overload of the aortic root can 
Table 22. Comparison between unrepaired TOF patients with dilatation and those without dilation as regard MDCT measurement of aortic root \&corresponding Z score.

\begin{tabular}{|c|c|c|c|}
\hline Characteristics & Dilated & Not dilated & p value \\
\hline \multicolumn{4}{|l|}{ Aortic annulus } \\
\hline Mean (SD) & 24.63 (3.99) & $13.2(2.62)$ & \multirow{2}{*}{$<0.0001^{*}$} \\
\hline Median (range) & $25(15-35)$ & $13(9-17)$ & \\
\hline \multicolumn{4}{|l|}{ Z score } \\
\hline Mean (SD) & 3.97 (0.85) & $0.93(0.51)$ & \multirow{2}{*}{$<0.0001^{*}$} \\
\hline Median (range) & $3.71(3.03-6.26)$ & $0.7(0.33-1.68)$ & \\
\hline \multicolumn{4}{|l|}{ STJ } \\
\hline Mean (SD) & $27.2(4.26)$ & $14.53(2.90)$ & \multirow{2}{*}{$<0.0001^{*}$} \\
\hline Median (range) & $27(17-40)$ & $14(10-19)$ & \\
\hline \multicolumn{4}{|l|}{ Z score } \\
\hline Mean (SD) & $3.09(0.62)$ & $2.07(5.26)$ & \multirow{2}{*}{$<0.0001^{*}$} \\
\hline Median (range) & $2.8(2.56-5.04)$ & $0.7(0.19-21)$ & \\
\hline \multicolumn{4}{|l|}{ Sinus of valsalva } \\
\hline Mean (SD) & 35.97 (4.59) & $20.53(3.40)$ & \multirow{2}{*}{$<0.0001^{*}$} \\
\hline Median (range) & $36(24-45)$ & $21(14-25)$ & \\
\hline \multicolumn{4}{|l|}{ Z score } \\
\hline Mean (SD) & $4.31(0.59)$ & $2.04(0.38)$ & \multirow{2}{*}{$<0.0001^{*}$} \\
\hline Median (range) & $4.3(3.24-5.66)$ & $2.02(0.99-2.46)$ & \\
\hline \multicolumn{4}{|l|}{ MAPCS/PDA } \\
\hline No & $16(45.71)$ & 15 (100.00) & \multirow{2}{*}{$<0.0001^{*}$} \\
\hline+ & 19 (54.29) & $0(0.00)$ & \\
\hline
\end{tabular}

cause aortic root dilatation in adults with a repaired TOF (2). Another causative mechanism for progressive aortic root dilatation is marked histological abnormalities in the aortic root and ascending aortic wall [26]. Progressive dilatation of the aortic root during long-term follow-up has also frequently been described after TOF repair; ranging in incidence between $15 \%$ - 88\% depending on definition of aortic root dilatation [26]. Our study demonstrated a dilated aortic root in 11 patients after surgical repair of Tetralogy of Fallot (TOF) representing 22\% of the study population. Aortic root measurements and the derived $\mathrm{Z}$ scores were $>3$ at the level of both aortic annulus and sinus of valsalva, with a $\mathrm{Z}$ score $>2.5$ at the level of sinotubular junction. The optimal definition for aortic root dilatation remains controversial. Normal aortic size is determined by age, sex, body size, location of the measurement, and robustness of the imaging modality. Consequently, normograms and $\mathrm{z}$ scores that adjust for age and body size are thought to more accurately identify aortic root dilatation [27]. In 2002 Niwa K reported a series of 32 patients who demonstrated a dilated aortic root after surgical repair of Tetralogy of Fallot [2]. Aortic root size measured by echocardiography was $>1.5$ expected diameter by standard nomogram. This "dilator" group comprised almost $15 \%$ of their TOF patient population. Characteristics of these patients with TOF compared with age-matched controls included a higher prevalence of pulmonary atresia, a longer shunt-torepair interval, and a right aortic arch. Other features were consequent on the dilated aorta rather than causal, moderate-to-severe aortic regurgitation and a larger left ventricle by echocardiography. Moreover, there was no significant difference in age at repair, duration of follow-up from repair, and time from the first to the last echo study between dilator and non-dilator TOF patients. Pulmonary atresia and a right aortic arch were more common in dilator group. Dilators had a longer time interval from palliation to repair and a greater root size markedly increased late after TOF repair in dilators compared with TOF controls. However, in our study, repaired dilator TOF patients showed statistically significant older age at repair with a p value of 0.03 , thus younger age at repair may abrogates late aortic root dilatation. This result is considered concordant to that done by Bhat et al., who reported that repair of TOF in infancy is associated with normalization of aortic root size in mid childhood with continuing normalcy into later childhood, whereas repair after infancy allows the persistence of marked aortic dilatation [28]. Also he concluded that early repair obviates the need for palliative shunts with their attendant complications, such as pulmonary artery distortion and aortic root dilatation [28]. Thus early volume unloading of aortic outflow with definitive repair may avoid progressive aortic root dilatation [28]. Rao BNS et al., 
in one publication reported no gender predominance among patients with TOF [29]. In contrast, there was a clear male predominance among patients with dilated aortic root even after indexing for body surface area and adapting for age according to Roman et al. [30]. In our study, although there was male predominance in the dilated repaired group being eight patients out of eleven, this was not statistically significant with a p value of 0.5 . Niwa k et al., reported high incidence of right aortic arch 50\% among dilator repaired TOF patients compared to non-dilator patients $26 \%$. In our study 54\% was the incidence of right aortic arch among dilator repaired TOF patients compared to $23 \%$ among non-dilators with statistical significant correlation between right arch and aortic root dilation with a p value of 0.04 . Also Niwa k et al., demonstrated $19 \%$ of the dilator repaired TOF patients had conduit repair for pulmonary atresia while none among the non-dilator group [31]. The result of our study is concordant to him with $18 \%$ of the dilator repaired TOF patients had conduit repair for pulmonary atresia while none among the non-dilator group. Moreover, Niwa k et al., reported $9 \%$ incidence of residual ventricular septal defect among dilator repaired TOF patients in his study while 4\% among the non-dilator group [31]. Compared to our study, residual ventricular septal defect was detected in $36 \%$ in the dilator repaired patients which was 4 fold higher than the previous study, also there was no residual ventricular septal defect detected in the non-dilator group with significant statistical correlation between residual VSD shunt and aortic root dilatation. In a recent study done by Nagy et al., on 2012, he concluded that, aortic regurgitation (AR), residual ventricular septal defect (VSD), and TOF with pulmonary atresia (TOF/PA) were associated with dilated aortic root and that sinus of Valsalva dilation after TOF repair is common and persists with aging [32]. Residual right ventricular outflow tract obstruction can persist after initial corrective surgery due to hypertrophied muscle in the subvalvular region, annular hypoplasia, pulmonary valve stenosis, or branch pulmonary artery stenosis [33]. Niwa K, in his study found association between absent pulmonary valve and aortic root dilatation, the incidence of absent pulmonary valve was $9 \%$ among dilator repaired TOF patients while zero among the non dilator group [34]. However in our study, a single patient with absent pulmonary valve was only found among dilator group, but there was no association to aortic dilatation due to lack of cases in our repaired patients. In our study dilated repaired group had significant lower mean\& median $\mathrm{O}_{2}$ saturation in comparison to non-dilated group therefore; correcting hypoxemia could protect against continuous development of aortic dilatation [35]. According to Chong WY, et al. 2006, aortic regurgitation was present in $12 \%$ of patients who tended to have larger $\mathrm{Z}$ scores at all aortic levels [36]. Aortic regurgitation is probably secondary to aortic root dilation as suggested by the greater Z scores of aortic dimensions in patients with aortic regurgitation compared to those without aortic regurgitation. In a second study done by Bhat, et al. on 2004, mild aortic regurgitation was reported in $23 \%$ of infancy repaired group, whereas more than mild aortic regurgitation was observed in $18 \%$ of the post-infancy repaired group [28]. In our study, however less than moderate aortic regurgitation was observed in $16 \%$ of repaired TOF patients who had larger Z scores at all aortic levels which was concordant to the above mentioned studies. Furthermore, aortic regurgitation secondary to aortic root dilatation occurred both in patients with repaired TOF with either pulmonary stenosis or pulmonary atresia [9]. In a study done by Chong et al., reported aortic root dilation in 67 children after repair of TOF; aortic dilation defined as a Z-score more than 2 was found in $88 \%$ at the annulus and $87 \%$ at the sinus of valsalva. Duration of follow-up was the only determinant of dilation; aortic regurgitation was present in $12 \%$ of patients who had larger dimensions at all aortic levels. These patients had increased aortic stiffness and reduced strain and distensibility. In this study TOF repair was performed at 3 years on average, and despite relatively early correction, the cases showed progressive aortic dilation. He has considered aortic dilation after TOF repair to be caused by the large right-to-left shunting occurring over many years before repair [36]. Francois K. et al., is the first to show on a larger data set, a regression of the aortic root diameters at different levels, with fastest size regression at the level of the aortic annulus and STJ, specifically during the first 3 years after repair. The size decrease in the aortic sinus appears to be slower but consistent, as proven by a significant decrease of indexed sinus diameter in most patients at the latest follow up [23]. In our study, larger mean and median Z scores values were recorded at the level of sinus of valsalva in comparison to the level of the aortic annulus and STJ values.

\subsection{Aortic Root Dilatation in Unrepaired Tetralogy of Fallot}

The first case report of progressive ascending aortic dilation in patients with TOF was published in early 1970s [37]. Chowdhury et al., reported aortic diameter measured by echocardiography showed significant dilatation in $66.3 \%$ of unrepaired patients with TOF in one publication and $68.9 \%$ in another publication [38]. In our study, 
aortic root diameter measured by MDCT showed significant dilation in $70 \%$ of unrepaired TOF patients. In Mayo Clinic series, the largest aortic root measured was $8.5 \mathrm{~cm}$ in diameter without dissection. The literature documents two isolated reports of aortic dissection with aortic root diameters of $6.45 \mathrm{~cm}$ [39] and $6.1 \mathrm{~cm}$ [40]. In our study the largest aortic root measured was $5.6 \mathrm{~cm}$ in diameter without dissection at the level of sinus of valsalva in uncorrected TOF patients. Two hypotheses have been postulated to explain this dilation. Firstly, increased blood flow from both ventricles to the overriding aorta before surgical repair is thought to be an underlying pathogenic mechanism, posing increased stress on the aortic wall [31]. Secondly, histological changes of the aortic media resembling those observed in patients with Marfan syndrome and bicuspid aortic valve So-called "cystic medial necrosis" is a misnomer because necrosis is seldom encountered and there are no true cysts; nonetheless, there is a non-inflammatory loss of smooth muscle cells, mucoid degeneration, and fragmentation of the elastic fibers within the media [34]. Incidence of male sex was $86.2 \%$ among TOF patients with dilated aorta [38]. In our study, we reported 85.71\% incidence of male sex among TOF patients with dilated aorta which was statistically significant. Aortic elasticity and distensibility are known to decline with age; these changes occur earlier and are accelerated among men [41]. Moreover, aortic stiffening precedes aortic dilation and predicts progressive aortic dilation, indicating that aortic stiffness plays a causative role in aortic dilation [42] [43]. This may be applicable for TOF patients and may explain in part the male predominance among dilator aortic group. Chronic hypoxia, is inevitable in patients with TOF before surgical correction, this has been shown to generate growth factors and matrix proteins that can induce irreversible vascular remodeling, smooth muscle proliferation and fibrosis [44]. In our study we reported significant statistical correlation between systemic arterial desaturation $<80 \%$ and aortic root dilation in unrepaired patients. On the other hand Bhat et al., 2004, reported no correlation between systemic saturation and aortic root size. Conceivably, aortic root dilatation is proportional to pulmonary stenosis (developmental basis). Additionally, antegrade flow across the aortic root increases proportionately to the severity of pulmonary outflow obstruction (hemodynamic basis) [28]. However, using systemic saturation as an index of pulmonary outflow obstruction in TOF may be a simplification that does not address multiple levels of obstruction or the presence of collateral blood supply. In a previous study conducted in 2002 by Niwa et al., the high incidence of right aortic arch (50\%) and pulmonary atresia (19\%) in their patients with marked aortic root dilatation and the relatively common coexistence of absent pulmonary valve syndrome may suggest a possible link between aortic root dilatation and chromosome 22 q11deletion. However, this was purely speculative and they did not have data on phenotypes or chromosomal analysis for their patients [2]. Right aortic arch has been reported in $25 \%$ of patients with TOF and is more common in patients with TOF and pulmonary atresia [45]. Furthermore, right aortic arch, pulmonary atresia, or absent pulmonary valve syndrome are common morphological features among TOF patients with 22q11 deletion [46]. We found statistically significant correlation between right arch and aortic root dilatation in our study, right arch has been reported in $28 \%$ of our TOF patients before intra-cardiac repair and in $37.14 \%$ in those with dilated aorta. Marelli AJ et al. 1994, substantiate suggestions of progressive right ventricular outflow tract obstruction in unrepaired TOF, with the extreme form being pulmonary atresia, increases right to left shunt through the ventricular septal defect and in turn the volume overloaded may contribute to aortic root dilatation [47]. In our study statistical significant higher RVOT gradients were measured among unrepaired TOF patients with dilated aortic root than those without root dilatation meanwhile significant lower PASP values were measured among unrepaired TOF patients with dilated aortic root than those without root dilatation. Chowdhury et al., demonstrated the presence of MAPCAs in (52.9\%) of 51 patients with aortic root dilatation. Medionecrosis, one of the histological abnormalities of the aortic media in dilated aorta was more prevalent in patients with presence of MAPCAs [38]. In our study 54.29\% of 35 patients with aortic root dilatation had MAPCs and or PDA. Aortic regurgitation in Tetralogy of Fallot imposes volume overload on both ventricles, but more importantly on the right ventricle which also confronts systemic afterload [2]. Although, initial palliative surgery involving arterial shunts (e.g. Blalock-Taussig shunt) would invariably result in greater aortic volume overloading with increased hemodynamic stress to the aortic wall, this was not identified as a significant determinant in a recent study [36]. In unrepaired TOF patients, AR is progressive and has been attributed primarily to lack of support of the aortic valve cusps, which prolapse into the sub-aortic VSD [48]. In addition, there is often dilation of the aortic annulus because the aorta receives most or all of the blood from both ventricles and this can contribute to aortic regurgitation. Other causes of aortic regurgitation in these patients include a congenitally abnormal aortic valve and damage from endocarditis [49]. In our study $25.71 \%$ among the dilator unrepaired TOF patients had up to mild 
AR, which represent a significant statistical correlation between AR and aortic root dilation with a p value of 0.03. Therefore, aortic dilatation and increased aortic stiffness due to aortic wall pathology in TOF patients may have a contributive effect on the development of AR and LV dysfunction, in addition to adverse right-to-left ventricular interaction [50]. Chowdhury et al. reported (60.7\%) incidence of aortic dilatation in patients with double outlet right ventricle (DORV) or TOF with pulmonary atresia; he demonstrated a relationship between aortic root dilatation and the presence of DORV [38]. In our study (62.16\%) of unrepaired dilator TOF patients had double outlet right ventricle (DORV) or TOF with pulmonary atresia, also we demonstrated a significant correlation between aortic root dilatation and the presence of DORV. However, Tan JL et al., observed no correlation between grade 2 or 3 histological changes in the aortic wall and pulmonary atresia, male sex, right aortic arch, or previous palliative surgery [26]. Another potential explanation for aortic root dilatation in patients with pulmonary atresia includes the disproportionate sharing of conotruncal tissue between aorta and pulmonary artery [51]. Moreover, Chowdhury reported $78.4 \%$ of patients with significant aortic root dilatation had aortic override greater than $50 \%$, whom they were found to be at increased risk of medionecrosis, development of fibrosis and muscle disarray in the aortic media predisposing to aortopathy [38]. In our study $57.14 \%$ of unrepaired dilator TOF patients had aortic override greater than $50 \%$ with statistically significant interrelationship between aortic root dilation and the degree of aortic overriding to the subaortic ventricular septal defect.

\section{Limitations}

The principal limitation of this study is its small sample size. Large number of patients is required to determine the extent of this problem and its correlation with various clinical entities with longer period of observation in future studies. The second limitation is that data on aortic root size before and early after TOF repair were not available. Thirdly, the echo studies were performed by different echocardiographers; therefore, inter- and intra-observer variability could not be assessed. In addition, 22q11 mutation status was not assessed in this cohort. Lastly, as patients with a dilated aorta were more likely to be followed up often, it is possible that the study suffers from ascertainment bias. This effect may be partly responsible for the apparent significant increase in $\mathrm{Z}$ score over time.

\section{Conclusion}

Male sex, previous conduit repair of pulmonary artesia, right aortic arch, systemic arterial desaturation, older age at repair, longer shunt to repair interval, residual post operative VSD shunt, impaired LV systolic function, presence of aortic regurgitation, pulmonary regurgitation, severe right ventricular outflow tract obstruction, TOF with pulmonary atresia, aortic override greater than 50\%, major aortopulmonary collaterals and/or PDA were independent risk factors for aortic root dilatation in Tetralogy of Fallot. These risk factors account for or may coexist with the higher incidence of aortic root dilatation encountered in subset of patients with TOF subjected to long-standing cyanosis and volume overload.

\section{References}

[1] Therrien, J. and Webb, G. (2003) Clinical Update on Adults with Congenital Heart Disease. The Lancet, 362, 13051313. http://dx.doi.org/10.1016/S0140-6736(03)14574-6

[2] Niwa, K., Siu, S.C., Webb, G.D. and Gatzoulis, M.A. (2002) Progressive Aortic Root Dilatation in Adults Later after Repair of Tetralogy of Fallot. Circulation, 106, 1374-1378. http://dx.doi.org/10.1161/01.CIR.0000028462.88597.AD

[3] McMahon, C.J., Ravekes, W.J. and Smith, E.O. (2004) Risk Factors for Neo-Aortic Root Enlargement and Aortic Regurgitation Following Arterial Switch Operation. Pediatric Cardiology, 25, 329-335. http://dx.doi.org/10.1007/s00246-003-0483-6

[4] Tzemos, N., Lyseggen, E., Silversides, C., Jamorski, M., Tong, J.H., Harvey, P., et al. (2010) Endothelial Function, Carotid-Femoral Stiffness, and Plasma Matrix Metalloproteinase-2 in Men with Bicuspid Aortic Valve and Dilated Aorta. Journal of the American College of Cardiology, 55, 660-668. http://dx.doi.org/10.1016/j.jacc.2009.08.080

[5] Oliver, J.M., Gallego, P., Gonzalez, A., Aroca, A., Bret, M. and Mesa, J.M. (2004) Risk Factors for Aortic Complications in Adults with Coarctation of the Aorta. Journal of the American College of Cardiology, 44, 1641-1647. http://dx.doi.org/10.1016/j.jacc.2004.07.037

[6] Ono, M., Goerler, H., Boethig, D., Westhorr-Bleck, M., Hartung, D. and Breymann, T. (2007) Valve-Sparing Operation for Aortic Root Aneurysm Late after Mustard Procedure. The Annals of Thoracic Surgery, 83, 2224-2226. 
http://dx.doi.org/10.1016/j.athoracsur.2006.12.043

[7] Lillehei, C.W., Varco, R.L., Cohen, M., Warden, H.E., Gott, V.L., DeWall, R.A., Patton, C. and Moller, J.H. (1986) The First Open Heart Corrections of Tetralogy of Fallot: A 26-31 Year Follow-Up of 106 Patients. Annals of Surgery, 204, 490-502. http://dx.doi.org/10.1097/00000658-198610000-00017

[8] Capelli, H., Ross, D. and Somerville, J. (1982) Aortic Regurgitation in Tetrad of Fallot and Pulmonary Atresia. American Journal of Cardiology, 49, 1979-1983. http://dx.doi.org/10.1016/0002-9149(82)90218-1

[9] Dodds, G.A., Warnes, C.A. and Danielson, G.K. (1997) Aortic Valve Replacement after Repair of Pulmonary Atresia and Ventricular Septal Defect or Tetralogy of Fallot. The Journal of Thoracic and Cardiovascular Surgery, 113, 736741. http://dx.doi.org/10.1016/S0022-5223(97)70232-0

[10] Marelli, A.J., Perloff, J.K., Child, J.S. and Laks, H. (1994) Pulmonary Atresia with Ventricular Septal Defect in Adults. Circulation, 89, 243-251. http://dx.doi.org/10.1161/01.CIR.89.1.243

[11] Anitha, S.J., Rychik, J., Yang, W., et al. (2009) 22q11.2 Deletion Status and the Risk of Aortic Root Dilation in Pediatric Patients with Tetralogy of Fallot. Circulation, 120, S585-S586.

[12] Sahn, D.J., Demaria, A., Kisslo, J., et al. (1978) Recommendations Regarding Quantitation in M-Mode Echocardiography: Results of a Survey of Echocardiographic Measurements. Circulation, 58, 1072-1083. http://dx.doi.org/10.1161/01.CIR.58.6.1072

[13] Broberg, C.S., Aboulhosn, J., Mongeon, F.P., Kay, J., Valente, A.M., Khairy, P., Earing, M.G., Opotowsky, A.R., Lui, G., Gersony, D.R., Cook, S., Ting, J.G., Webb, G. and Gurvitz, M.Z. (2011) Alliance for Adult Research in Congenital Cardiology (AARCC). Prevalence of Left Ventricular Systolic Dysfunction in Adults with Repaired Tetralogy of Fallot. American Journal of Cardiology, 107, 1215-1220. http://dx.doi.org/10.1016/j.amjcard.2010.12.026

[14] Zoghbi, W.A., Enriquez-Sarano, M., Foster, E., Grayburn, P.A., Kraft, C.D., Levine, R.A., Nihoyannopoulos, P., Otto, C.M., Quinones, M.A., Rakowski, H., Stewart, W.J., Waggoner, A., Weissman, N.J. (2003) American Society of Echocardiography. Recommendations for Evaluation of the Severity of Native Valvular Regurgitation with Two-Dimensional and Doppler Echocardiography. Journal of the American Society of Echocardiography, 16, 777-802. http://dx.doi.org/10.1016/S0894-7317(03)00335-3

[15] Suzuki, Y., Kambara, H., Kadota, K., et al. (1986) Detection and Evaluation of Tricuspid Regurgitation Using a RealTime, Two-Dimensional, Color-Coded, Doppler Flow Imaging System: Comparison with Contrast Two-Dimensional Echocardiography and Right Ventriculography. American Journal of Cardiology, 57, 811-815. http://dx.doi.org/10.1016/0002-9149(86)90619-3

[16] Williams, R.V., Minich, L.L., Shaddy, R.E., et al. (2002) Comparison of Doppler Echocardiography with Angiography for Determining the Severity of Pulmonary Regurgitation. American Journal of Cardiology, 89, 1438-1441. http://dx.doi.org/10.1016/S0002-9149(02)02365-2

[17] Sahn, D.J., DeMaria, A., Kisslo, J., et al. (1978) Recommendations Regarding Quantitation in M-Mode Echocardiograpy: Results of a Survey of Echocardiographic Measurements. Circulation, 58, 1072-1083. http://dx.doi.org/10.1161/01.CIR.58.6.1072

[18] Yang, M., Mo, X.M., Jin, J.Y., Wu, M., Liu, B., Liu, Z.Y., Gao, X.C., Tang, W.W. and Teng, G.J. (2010) Diagnostic Value of 64 Multislice CT in Typing of Congenital Aortic Anomaly in Neonates and Infants. National Medical Journal of China, 90, 2167-2171.

[19] Bailliard, F., Hughes, M.L. and Taylor, A.M. (2008) Introduction to Cardiac Imaging in Infants and Children: Techniques, Potential, and Role in the Imaging Workup of Various Cardiac Malformations and Other Pediatric Heart Conditions. European Journal of Radiology, 68, 191-198. http://dx.doi.org/10.1016/j.ejrad.2008.05.016

[20] Tops, L.F., Wood, D.A., Delgado, V., et al. (2008) Noninvasive Evaluation of the Aortic Root with Multislice Computed Tomography. JACC: Cardiovascular Imaging, 1, 321-330. http://dx.doi.org/10.1016/j.jcmg.2007.12.006

[21] Loren, F., George, L., Joshua, A., et al. (2010) Recommendations for Aortic Imaging Techniques to Determine the Presence and Progression of Thoracic Aortic Disease, Guidelines for the Diagnosis and Management of Patients with Thoracic Aortic Disease: Executive Summary. Circulation, 3, 14-17.

[22] Pettersen, M.D., Du, W., Skeens, M.E. and Humes, R.A. (2008) Regression Equations for Calculation of Z Scores of Cardiac Structures in a Large Cohort of Healthy Infants, Children, and Adolescents: An Echocardiographic Study. Journal of the American Society of Echocardiography, 8, 922-934. http://dx.doi.org/10.1016/j.echo.2008.02.006

[23] François, K., Zaqout, M., Bové, T., et al. (2010) The Fate of the Aortic Root after Early Repair of Tetralogy of Fallot. European Journal Cardio-Thoracic Surgery, 37, 1254-1258.

[24] Stulak, J.M., Dearani, J.A., Burkhart, H.M., Sundt, T.M., Connolly, H.M., Schaff, H.V. (2010) Does the Dilated Ascending Aorta in an Adult with Congenital Heart Disease Require Intervention? The Journal of Thoracic and Cardiovascular Surgery, 140, S52-S57. http://dx.doi.org/10.1016/j.jtcvs.2010.08.052

[25] Matsuoka, R., Takao, A., Kimura, M., et al. (1994) Confirmation that the Conotruncal Anomaly Face Syndrome Is 
Associated with Deletion within 22q11.2. American Journal of Medical Genetics, 53 285-289. http://dx.doi.org/10.1002/ajmg.1320530314

[26] Tan, J.L., Davlouros, P.A., McCarthy, K.P., Gatzoulis, M.A. and Ho, S.Y. (2005) Intrinsic Histological Abnormalities of Aortic Root and Ascending Aorta in Tetralogy of Fallot: Evidence of Causative Mechanism for Aortic Dilatation and Aortopathy. Circulation, 112, 961-968. http://dx.doi.org/10.1161/CIRCULATIONAHA.105.537928

[27] Hiratzka, L.F., Bakris, G.L., Beckman, J.A., Bersin, R.M., Carr, V.F., Casey Jr., D.E., Eagle, K.A., Hermann, L.K., Isselbacher, E.M., Kazerooni, E.A., Kouchoukos, N.T., Lytle, B.W., Milewicz, D.M., Reich, D.L., Sen, S., Shinn, J.A., Svensson, L.G. and Williams, D.M. (2010) Guidelines for the Diagnosis and Management of Patients with Thoracic Aortic Disease: A Report of the American College of Cardiology Foundation/American Heart Association Task Force on Practice Guidelines, American Association for Thoracic Surgery, American College of Radiology, American Stroke Association, Society of Cardiovascular Anesthesiologists, Society for Cardiovascular Angiography and Interventions, Society of Interventional Radiology, Society of Thoracic Surgeons, and Society for Vascular Medicine. Circulation, 121, e266-e369.

[28] Bhat, A.H., Smith, C.J. and Hawker, R.E. (2004) Late Aortic Root Dilatation in Tetralogy of Fallot May Be Prevented by Early Repair in Infancy. Pediatric Cardiology, 25, 654-659. http://dx.doi.org/10.1007/s00246-003-0665-2

[29] Rao, B.N.S., Anderson, R.C. and Edwards, J.E. (1971) Anatomic Variations in the Tetralogy of Fallot. American Heart Journal, 81, 361-371. http://dx.doi.org/10.1016/0002-8703(71)90106-2

[30] Roman, M.J., Devereux, R.B., Kramer-Fox, R., et al. (1989) Two-Dimensional Echocardiographic Aortic Root Dimensions in Normal Children and Adults. American Journal of Cardiology, 64, 507-512. http://dx.doi.org/10.1016/0002-9149(89)90430-X

[31] Niwa, K. (2005) Aortic Root Dilatation in Tetralogy of Fallot Long-Term after Repairhistology of the Aorta in Tetralogy of Fallot: Evidence of Intrinsic Aortopathy. International Journal of Cardiology, 103, 117-119. http://dx.doi.org/10.1016/j.ijcard.2004.07.002

[32] Nagy, C.D., Alejo, D.E., Corretti, M.C., et al. (2013) Tetralogy of Fallot and Aortic Root Dilation: A Long-Term Outlook. Pediatric Cardiology, 34, 809-816.

[33] Hennein, H.A., Mosca, R.S., Urcelay, G., et al. (1995) Intermediate Results after Complete Repair of Tetralogy of Fallot in Neonates. The Journal of Thoracic and Cardiovascular Surgery, 109, 332-344. http://dx.doi.org/10.1016/S0022-5223(95)70395-0

[34] Niwa, K., Perloff, J.K., Bhuta, S.M., et al. (2001) Structural Abnormalities of Great Arterial Walls in Congenital Heart Disease: Light and Electron Microscopic Analyses. Circulation, 103, 393-400. http://dx.doi.org/10.1161/01.CIR.103.3.393

[35] Lee, L.C., Torres, M.C., Khoo, S.M., et al. (2010) The Relative Impact of Obstructive Sleep Apnea and Hypertension on the Structural and Functional Changes of the Thoracic Aorta. Sleep, 33, 1173-1176.

[36] Chong, W.Y., Wong, W.H., Chiu, C.S. and Cheung, Y.F. (2006) Aortic Root Dilation and Aortic Elastic Properties in Children after Repair of Tetralogy of Fallot. American Journal of Cardiology, 97, 905-909. http://dx.doi.org/10.1016/j.amjcard.2005.09.141

[37] Peters, R.M. (1971) Total Repair of Tetralogy of Fallot and Replacement of Aortic Valve with Starr-Edwards Prosthesis. Case Report. The Journal of Thoracic and Cardiovascular Surgery, 61, 599-601.

[38] Chowdhury, U.K., Mishra, A.K., Ray, R., et al. (2008) Histopathological Changes in Ascending Aorta and the Risk Factors Related to Histopathological Conditions and Aortic Dilatation in Patients with Tetralogy of Fallot. The Journal of Thoracic and Cardiovascular Surgery, 135, 69-77. http://dx.doi.org/10.1016/j.jtcvs.2007.06.011

[39] Kim, W.H., Seo, J.W., Kim, S.J., et al. (2005) Aortic Dissection Late after Repair of Tetralogy of Fallot. International Journal of Cardiology, 101, 515-516. http://dx.doi.org/10.1016/j.ijcard.2004.03.026

[40] Rathi, V.K., Doyle, M., Williams, R.B., et al. (2005) Massive Aortic Aneurysm and Dissection in Repaired Tetralogy of Fallot; Diagnosis by Cardiovascular Magnetic Resonance Imaging. International Journal of Cardiology, 101, 169170. http://dx.doi.org/10.1016/j.ijcard.2004.05.037

[41] Sonesson, B., Lanne, T., Vesnersson, E., et al. (1994) Sex Difference in the Mechanical Properties of the Abdominal Aorta in Human Being. Journal of Vascular Surgery, 20, 959-965. http://dx.doi.org/10.1016/0741-5214(94)90234-8

[42] Nollen, G.J., Groenink, M., Tijssen, J.G., Van Der Wall, E.E. and Mulder, B.J. (2004) Aortic Stiffness and Diameter Predict Progressive Aortic Dilatation in Patients with Marfan Syndrome. European Heart Journal, 25, 1146-1152. http://dx.doi.org/10.1016/j.ehj.2004.04.033

[43] Nistri, S., Grande-Allen, J., Noale, M., Basso, C., Siviero, P., Maggi, S., Crepaldi, G. and Thiene, G. (2008) Aortic Elasticity and Size in Bicuspid Aortic Valve Syndrome. European Heart Journal, 29, 472-479. http://dx.doi.org/10.1093/eurheartj/ehm528

[44] Faller, D.V. (1999) Endothelial Cell Responses to Hypoxic Stress. Clinical and Experimental Pharmacology and Phy- 
siology, 26, 74-84. http://dx.doi.org/10.1046/j.1440-1681.1999.02992.x

[45] Rao, B.N.S., Anderson, R.C. and Edwards, J.E. (1971) Anatomic Variations in the Tetralogy of Fallot. American Heart Journal, 81, 361-371. http://dx.doi.org/10.1016/0002-8703(71)90106-2

[46] Johnson, M.C., Strauss, A.W., Dawton, B., et al. (1995) Deletion within Chromosome 22 Is Common in Patients with Absent Pulmonary Valve Syndrome. American Journal of Cardiology, 76, 66-69. http://dx.doi.org/10.1016/S0002-9149(99)80803-0

[47] Marelli, A.J., Perloff, J.K., Child, J.S., et al. (1994) Pulmonary Atresia with Ventricular Septal Defect in Adults. Circulation, 89, 243-251. http://dx.doi.org/10.1161/01.CIR.89.1.243

[48] Bull, K., Somerville, J., Ty, E., et al. (1995) Presentation and Attrition in Complex Pulmonary Atresia. Journal of the American College of Cardiology, 25, 491-499. http://dx.doi.org/10.1016/0735-1097(94)00364-V

[49] Matsuda, H., Ihara, K., Mori, T., et al. (1980) Tetralogy of Fallot Associated with Aortic Insufficiency. The Annals of Thoracic Surgery, 29, 529-533. http://dx.doi.org/10.1016/S0003-4975(10)61699-2

[50] Geva, T., Sandweiss, B.M., Gauvreau, K., et al. (2004) Factors Associated with Impaired Clinical Status in Long-Term Survivors of Tetralogy of Fallot Repair Evaluated by Magnetic Resonance Imaging. Journal of the American College of Cardiology, 43, 1068-1074. http://dx.doi.org/10.1016/j.jacc.2003.10.045

[51] Anderson, R.H., Cook, A., Brown, N.A., Henderson, D.J., Chaudhry, B. and Mohun, T. (2010) Development of the Outflow Tracts with Reference to Aortopulmonary Windows and Aortoventricular Tunnels. Cardiology in the Young, 20, 92-99. http://dx.doi.org/10.1017/S1047951110001137 